\title{
The Immunohistochemical Expression of Bcl2, Ki 67and CD3 correlated with the Basal Cell Carcinoma Histological Features - a study on 10 cases
}

\author{
MONICA-GEORGIANA NICOLA ${ }^{1 *}$, LILIANA MOCANU ${ }^{2}$, ARITINA ELVIRA MOROSANU $^{3}$, \\ SORIN BERBECE ${ }^{4}$, LOREDANA ELENA STOICA ${ }^{5}$ \\ ${ }^{1}$ Ovidius University Constanta, Faculty of Medicine, Department of Dermatology, 124 Mamaia Blvd., 900527, \\ Constanța, Romania \\ ${ }^{2}$ University Emergency Country Hospital, Department of Pathology, 145 Tomis Blvd., 900591, Constanța, Romania \\ ${ }^{3}$ University of Medicine and Pharmacy of Craiova, Department of Pediatrics, 2 Petru Rares Str., 200349, Craiova, \\ Romania \\ ${ }^{4}$ Dunarea de Jos University, Department of Morphological and Functional Sciences, Faculty of Medicine and Pharmacy, \\ 47 Domneasca Str., 800008, Galati, Romania \\ ${ }^{5}$ University of Medicine and Pharmacy of Craiova, Department of Dermatology, 2 Petru Rares Str., 200349, Craiova, \\ Romania
}

The basal cell carcinoma represents a worldwide major health problem due to incidence, functional and aesthetical defects which accompanies the disease and its treatment and high recurrency risk.The various morphoclinical forms of the basal cell carcinoma may be explained due to at least three factors: genetical predisposal, the individual immunological status and the action of the risk factors. In the case in which the histological aspects do present some modifications which cannot be catalogued in the usual patterns, therefore inseriated sections of the hole tumor being imposed, in order to establish correctly the diagnosis and in the case in which microscopical criteria are partially overposable with those specific to another lesion, the immunohistochemical evaluation is imposed.The immunohistochemical studies are also used in research, thus revealing some biological aspects of the basal cell carcinoma. The study of the biological markers of the apoptosis, cellullarproliferation and the immunitary system reaction allows the understanding of the morphofunctional anomalies during the neoplastic transformation process and the early identification of the aggressive forms.

Keywords: basal cell carcinoma; Bcl2; Ki67; CD3

The basal cell carcinoma (BCC) is a form of non-melanocitary skin cancer [1]. Generally, it has a slow growing rhythm, but also presents aggressive forms, with deep tissular invasion. The non-aggressive forms do have a slow growth, thus including the nodular and superficial types. The aggressive forms (micronodular; morpheiform, sclerotic and metatypic) are predisposed to frequent recurrences. The BCC cannot be classified as invasive and non-invasive. The non-invasive or superficial type does not invade, or minimally invades the superficial dermal layers, the apoptotic cells being rare in the basaloid cellular buds [2]. The invasive form may be infiltrative (sclerogenous; nonsclerogenous) and non-infiltrative (nodular, micronodular). The infiltrative growth is characterized by irregularlyshaped cellular nests, of variable dimensions with sharp-angled, serrated, peripheral contours and non-net inferior tumoral edge [3].

The clinical aspect does not correspond with the histological one, inside the same tumor being possible to be present various histological types, having different behavior, reason why the clinical approach must be differentiated, according with the clinical case particularity. The intervention of the immunitary system on the BCC evolution can be interpreted by an architectural destruction of the tumor cells, by the appearance of the apoptotic cells and of the dermal collagen deposits, according to studies realized by Curson and Weedon [4].

The Bcl2, regulatory protein, codified on humans by the BCL2 gene, mostly located on the mitochondrial membrane, is a suppressor of the apoptosis. The suppression of the apoptosis by the stimulating agents of the tumor do represent one of the most important mechanisms in promoting the tumor. The tumor cell is loosing its memory. The apoptotic genes activation do represent a mechanism by which the immunitary system cannot intervene in the antitumoral protection, this way appearing an imbalance between the high cellular proliferation rate and the low cellular death rate $[5,6]$. The cellular proliferation and the apoptosis are important elements in the assembly of events which determine the transformation of a normal cell into a malignant one [7-9]. 
The Ki67 monoclonal antibody is reacting with a nuclear antigen which is present during the G1;S and G2 phases of the cellular cycle, thus being possible the detection of the growth fractions.

Another way by which the tumor cells are not destroyed by the immunitary system is the deficiency of costimulating molecules, with the determination of a low stimulation level of the T-cells proliferation, so that the local lymphocyte infiltrate is unable to fully activate itself in order to destroy the tumor cells. The CD3 molecule, discovered in 1979 by Kung and collaborators, is a component of the TCR-CD3 complex, with the role of activating the T-lymphocytes (cytotoxic and helper). Being present in all the cellular development stages, the CD3 do represent an immunohistochemical marker for the T lymphocytes [10,11,12].

The molecular biology data determining of every BCC form may help us, in the future, to establish a targeted treatment which could have as result the healing.

\section{Experimental part}

Materials and methods

The present study included 10 selected cases of BCC, randomly selected confirmed in the Pathological Anatomy Laboratory of the "St.Andrew" Clinical County Hospital of Constanta. The tumor samples were rapidly fixed in 10\% formalin (ischemic extracorporeal time-IECT-betweem5 and 10 minutes time). All the samples were processed by paraffin embedding and automatically mean (the DIAPATH platform). Five-micron sections were practiced and these were hematoxiline-eosine stained by conventional techniques (progressive method, DIAPATH).After reconfirmation of the diagnosis, immunohistochemical tests were realized by polymer method, with HRP/DAB visualization detection system, on BIOCARE semiautomatic platform, with following antibodies: Bcl2 (mAb clone bcl-2, Novocastra), Ki67 (MIB-1 clone, Dako) and CD3 (clone F7.2.38, Dako), respecting all producers protocols.

The immunohistochemical reaction was evaluated by its intensity: weak $(+)$, moderate $(++)$, intense $(+++)$. For certifying the immunohistochemical reactions, we used controls as it follows: for the Bcl2, we used the peritumoral B lymphocytes as internal positive control; Ki67 had as internal positive control the basal keratocytes, and for CD3, we used external control the palatine tonsil and the distribution was objectivated, like in melanoma, as "brisk" type (continuous at the tumor base), or "non-brisk" (discontinuous or even absent at the base of the tumor).

\section{Image analysis}

The images with the tumoral areas and the adjacent epidermis were photographed with a LEICA ICCHD 50 HD photo camera mounted on a LEICA DM 750 microscope using LAS V4.6 software, in constant and luminosity conditions.

\section{Results and discussions}

Histopathological exam revealed the following types: nodular solid with central necrosis type (2 cases) (Fig. 1), nodular solid without central necrosis type (3 cases) (Fig. 2), mixed nodular and infitrative types (1 case) (Fig. 3) and multicentric superficial (4 cases) (Fig. 4)

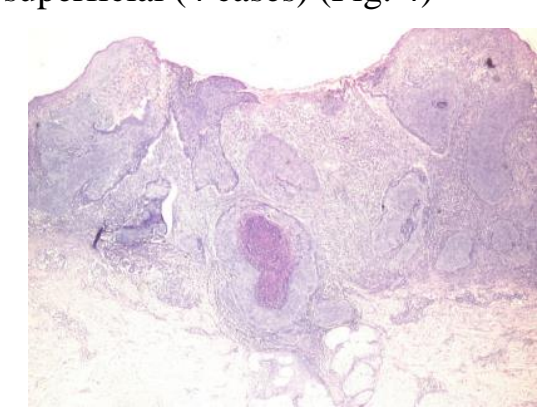

Fig. 1. BCC nodular solid type with central necrosis

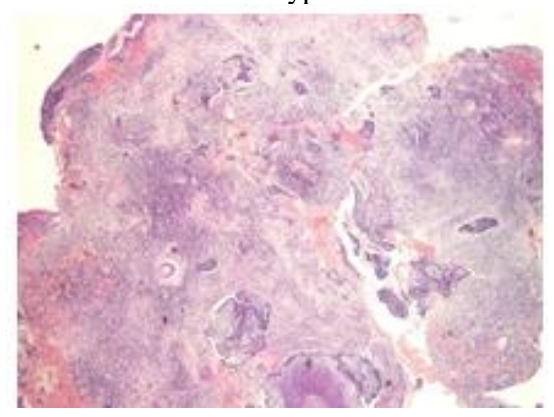

Fig.3. BCC mixed nodular and infitrative type

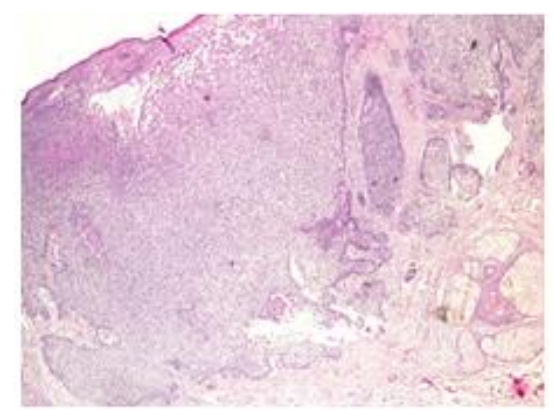

Fig. 2.BCC nodular solid type with central necrosis

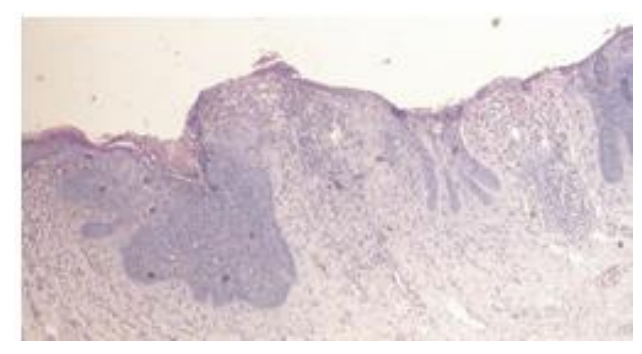

Fig.4. BCC multicentric superficial 
Bcl2 immunostaining The cytoplasmatic reaction and/or nuclear reaction on $\mathrm{Bcl} 2$ were variable. The infiltrative and superficial $\mathrm{BCC}$ had a maximal intensity cytoplasmatic reaction. The components of the collisional type reacted differently, the solid component having a weak reaction+ $(60 \%)$, comparing with the infiltrative component+++, as in the purely solid forms (Figure 5, 6). In a nodular with central necrosis-type BCC, we obtained a $++/+++(80 \%)$ and in the other case a negative reaction, but the internal control was unconvincing (Fig. 7). The multicentric superficial forms reacted $++/+++$, in an $80 \%$ cases (Fig. 8).

Ki67 immunostaining The reaction on Ki67 was generally intensely positive in variable proportion (10-40 percent), the maximal value being observed in the collisional solid-infiltrative form, with similar values in both components (Figure 9). Inside the solid non-infiltrative tumors was observed a depression of the reaction on Ki67 (Fig. 10).

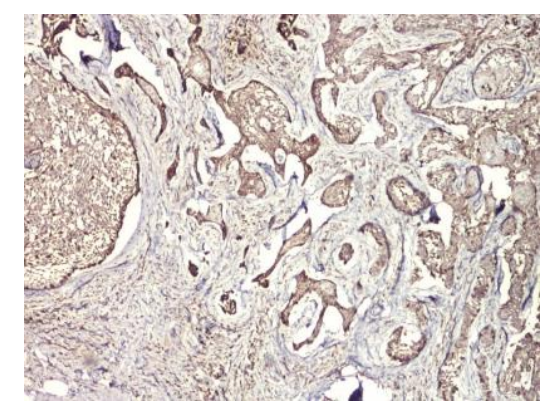

Fig.5. BCC nodular and infitrative type - $\mathrm{Bcl} 2+++$

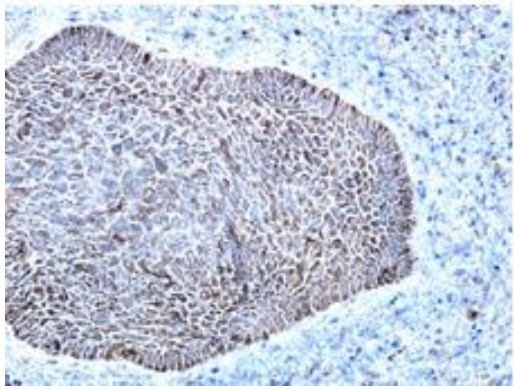

Fig.6. BCC nodular type - Bcl $2+$

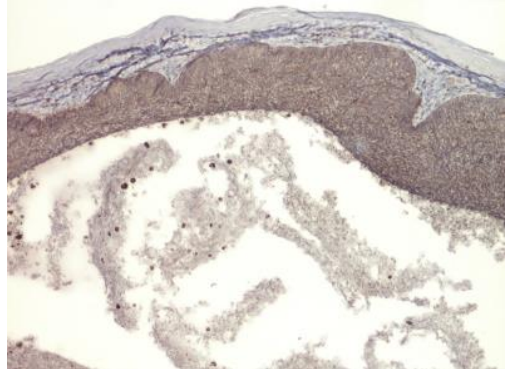

Fig.7. Nodular with central necrosis - type BCC - Bcl 2+++

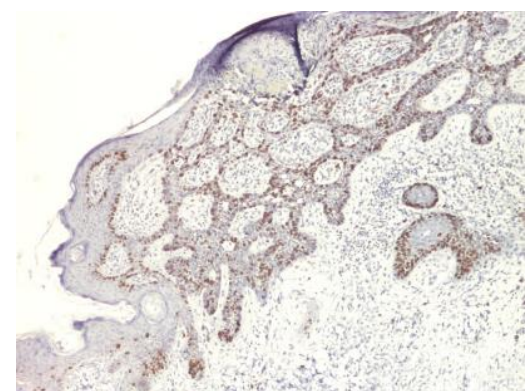

Fig.9. BCC infitrative type $-\mathrm{Ki67+++}$

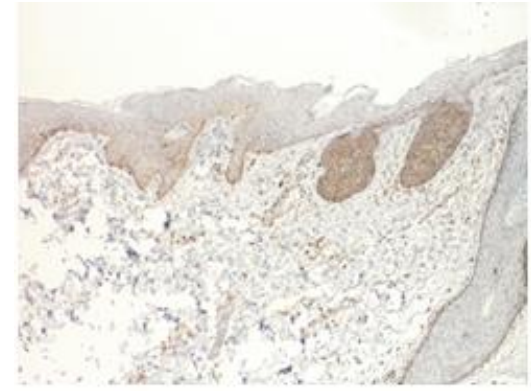

Fig.8. BCC superficial type - Bcl $2+++$

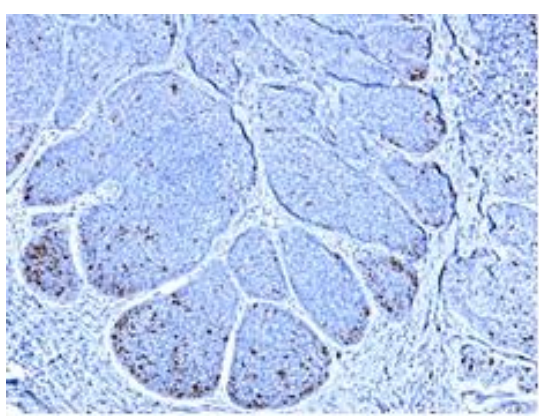

Fig.10 - Ki67+ in tumour cells

CD3 immunostaining The reaction on CD3 was intense+++, brisk-type in the case of the 35-years old female patient and non-brisk in the case of over-50-years old patients (Figs. 11, 12).

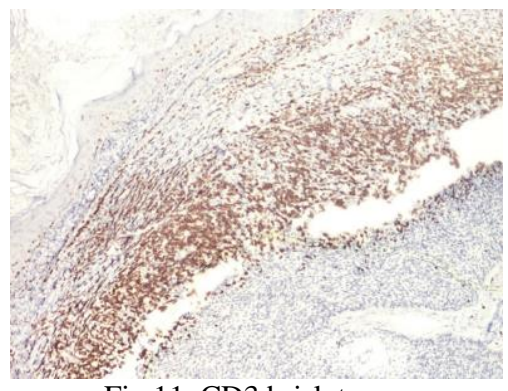

Fig.11. CD3 brisk type +++

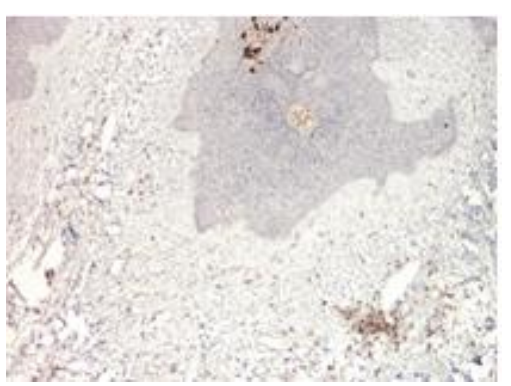

Fig.12. Noduar BCC - CD3 brisk type +++ 
$\mathrm{Bcl} 2$ is not present in the stem cells from the health tegument, but is present in the tumor cells, notably on BCC. The cytoarchitectural distribution of bcl-2 in the normal skin includes: the basal keratocytes, the dermal papilla of the hair follicle, the Huxley and Henle keratinized layers, the cells of the external keratinized layers of the isthmus and infundibulum of the hair follicle. The Bcl-2 is the antiapoptotic gene which promotes the cellular viability without influencing the cellular proliferation. The over-production of Bcl-2 partially suppresses the apoptosis induced by p53. The Bcl-2 oncoprotein is utilized in the differential diagnosis of the BCC comparing with trychoepitelioma and the actinic keratosis. In the BCC, all the tumor lobules do react positively and in the trychoepitelioma only the peripheral cells are expressing Bcl2 [13]. It is considered a aggressivity marker, so the aggressive BCCs, although positive to bcl2, do have a lower reactivity comparing with the non-aggressive ones. Our results are accordingly to data from specialty literature, the multicentric superficial form presents a higher reactivity to bcl-2, as the solid form presents a higher one.

The Ki67 non-histonic protein is a marker of cellular proliferation, being present in all active stages of the cellular cycle (besides G0), thus a significant correlation between the proliferation index and the mitotic activity. It has a variable behavior in the BCC and cannot be considered a severity marker [14]. Ki67 is intensely positive, but in variable proportions (data obtained by us are corresponding to those in the literature) is correlated with a slow evolution of the BCC. The difference between the values of the reaction to $\mathrm{Bcl} 2$ and the values to Ki67 demonstrates an ununiform proliferation; it is not enough that the apoptosis shall be stopped in order to allow the continuity of the cell proliferation.

The CD3 is expressed, on cytoplasmatic level, in the case of the NK cells, and on membrane level in the case of lymphocytes. The positive expression for $\mathrm{CD} 3$ confirmed the presence of the peritumoral inflammatory infiltrate in all cases. The distribution difference between brisk/non-brisk on the same form (multicentric superficial) may explain the different evolution. The evolution is influenced by the histological form, but the same form may evolve differently from patient to patient, accordingly to immunitary status. The interstitial-epithelial interactions do have a special importance, the incorrect regulation may stimulate tumorogenesis and invasion [15].

The inflammatory band-type infiltrate does not allow infiltrative development, producing an efficient regression mechanism or even tumor elimination by the host. According to data in the specialty literature, the age represents an important factor in the BCC evolution, but the small case number does not allow us to establish significant correlations. The immunitary status to a high age causes a low defenses reaction, allowing the tumoral cells to infiltrate. The melanoma with brisk-type reaction do have a survival rate from 5 to 10 years, superior comparing to non-brisk reaction cases or even absent [16]. The absence of the inflammatory infiltrate allows the tumor to infiltrate. The proliferation index is low in the BCC case. In the case of collisional BCC, the proliferation percentage of the solid form is high if it does also present an infiltrative component, comparing to the pure solid form. This fact could explain why it does reoccur, although the histopathological exam classified it into a non-aggressive form. The study demonstrates a different behavior of the tumoral mass according to histological structure. The in seriated sections of the hole tumor may help us establishing the tumoral behavior.

\section{Conclusions}

It is well known the fact that the immunitary system reacts in a special way in the case of the presence of a malignant tumor. By comparing the expression of all those markers that we have studied, we can identify the most aggressive types of BCC and asses their biological behavior. As for the BCC, there are not enough data in order to convince us about the presence of a certain reaction of the immunitary system.

Abbreviations used in the text:

BCC: basal cell carcinoma

TCR: T Cell Receptors

NK: Natural Killer

Bcl2: B-cell lymphoma 2

IECT: ischemic extra corporeal time

\section{References}

1.BOLOGNIA JL, JORIZZO JL, SCHAFFER JV. Dermatology, In Russell Gabbedy (ed) Third Edition, Elsevier Saunders, 2012, Elsevier Limited, Ebook ISBN 9780702051821, pp:1759-1773

2.CROWSON AN, MAGRO CM, KADIN M, ET AL. Diferential expression of bcl-2 oncogene in human basal cell carcinoma. Hum Pathol 1996; 27: 355-359

3.SIEGLE RJ, MAC MILLAN J, POLLACK SV. Infiltrative basal cell carcinoma: a nonsclerosing subtype. J Dermatol Surg Oncol 1986; $12: 830-8360$

4.CURSON C, WEEDON D Spontaneous regression in basal cell carcinomas J Cutan Pathol; 1979; 6(5):432-7

5.PARDAL R, MOLOFSKY AV, MORRISON SJ. Stem cell self-renewal and cancer cell proliferation are regulated by common networks that balance the activation of proto-oncogenes and tumor suppressors. Cold Spring Harb. Symp. Quant. Biol 70, 177-185 
6.SPILLANE JB, HENDERSON MA, Cancer stem cells: a review. ANZ. J.Surg. 2007, 77: 464-468

7.ADAMS JM, CORY S. The BCL-2 arbiters of apoptosis and their growing rol as cancer targets. Cell Death\&Differentiation 2018; 25, 27-36

8.WINTZER HO, ZIPFEL I, SCHULTE-MONTING J, HELLERICH U, VON KLEIST S. Ki-67 immunostaining in human breast tumors and its relationship to prognosis. Cancer. 1991; 67: 421-428

9.HASCHEK AND ROUSSEAUX's Handbook of Toxicologic Pathology. Third Edition. 2013, 107-146

10.VANDEBROEK A, SCHRJVERS D. Gene therapy. In Meestedt H, Schjvers D, Bafaloukos D, Greil R. European Socoety for Medical Oncology Handbook of principles of translational research. Informa Healthcare 2007: 121-127

11.HLADNIK LM, WIL AR, AUGUSTIN KM. Principles of systemic cancer therapy: moleculary targeted therapy. In Govondan R (ed): the Washington Manual of Oncology. Second edition, Wolter Kluwer/Lippincott Williams\&Wilkins, Philadelphia 2008: 33-46

12.CASCIATO DA. Cancer chemotherapy. In: Casciato DA (ed): Manual of clinical oncology. Sixth edition. WoltersKluver/Lippincott Williams\&Wilkins, Philadepphia 2009: 46-99

13.LE STOICA, RC DASCÂLU AND ALL Solitary trichoepithelioma: clinical, dermatoscopic and histopathological findings case report Rom J Morphol Embryol 2015, 56(2 Suppl):827-832

14.CORREA MDE P, FERREIRA AP, GOLLNER AM, RODRIGUES MF, GUERRA MC Markers expression of cell proliferation and apoptosis in basal cell carcinoma An Bras Dermatol. 2009 Nov-Dec;84(6):606-14.

15.PROVENZANO PP, ELICEIRI KW, CAMPBELL JM, INMAN DR, WHITE JG, KEELY PJ. Collagen reorganization at the tumor-stromal interface facilitates local invasion. BMC Medicine 2006, 4:38

16.CLEMENTE CG ET COL. Prognostic value of tumor infiltrating lymphocytes in the vertical growth phase of primary cutaneous melanoma. Cancer.1996 Apr 1; 77(7):1303-1307

Manuscript received: 10.07.2019 